\title{
EFFECT OF VISCOUS DAMPING IN NUMERICAL CALCULATION OF CABLE-MEMBRANE STRUCTURES BY THE METHOD OF DYNAMIC RELAXATION
}

\author{
Miloš Huttner*, Petr FAJMAn \\ Czech Technical University in Prague, Faculty of Civil Engineering, Thakurova 7, 16629 Prague 6, Czech \\ Republic \\ * corresponding author: milos.huttner@fsv.cvut.cz
}

\begin{abstract}
The aim of this article is to assess the speed of convergence of numerical calculation of cable-membrane structures using dynamic relaxation method in the process of finding critical damping pre-calculated on undamped system. The procedure is tested on four different constructions in six numerical cases. The variety of examples is as large as possible to demonstrate the greatest versatility of the test procedure. The efficiency of the procedure is evaluated based on the number of iterations.
\end{abstract}

KEYworDs: Cable-membrane structures, dynamic relaxation, critical damping, viscous damping.

\section{INTRODUCTION}

The design of cable-membrane structures is realized using numerical models of construction. Execution of the numerical analysis is not easy. The shape of the structure, unlike conventional structures, is not known in advance. The initial equilibrium shape of the structure must be found during the phase called the form-finding process. The second phase is the static response process. Both processes represent a geometrically non-linear case from a mathematical point of view, but each process has its own specifics.

Different matrix methods have been developed to address both processes (for example force density method [1], update reference strategy [2], transient stiffness method, etc.). The method of dynamic relaxation represents a very suitable alternative to matrix procedures [3]. In addition, the method can be easily used for both phases of the design of cablemembrane structures. Consuming CPU time with more unknowns takes approximately linearly, so the method is very well applicable to models with a large number of degrees of freedom [4].

The dynamic relaxation method builds on the principle that the structure under free oscillation is directed to static equilibrium. The fictitious dynamic analysis is used to find static equilibrium. The artificial parameters (masses, a damping factor, a time interval) influence the speed of convergence. The convergence is the fastest if the damping (a viscous damping is considered) is critical [4]. For fast convergence, it is necessary to find the fundamental oscillation frequency, which is not easy for geometrically non-linear cases. One possible way is to use a pre-calculation with an undamped oscillation mode.

The aim of this article is to assess the speed of convergence of numerical calculation of cable-membrane structures in the process of finding critical damping pre-calculated on undamped system. The procedure is tested on four different constructions in six numerical cases. Both design processes (form-finding and static response) will be included.

\section{Methodology}

Critical viscous damping coefficient can be found from [4]:

$$
C=4 \pi m f,
$$

where $C$ is viscous damping coefficient, $m$ is mass (or vector of masses), $f$ fundamental frequency of oscillation.

The fundamental frequency of oscillation can be obtained pre-calculated on undamped system, with $C$ set to zero. Based on the coordinate displacement record, the fundamental oscillation frequency is determined by the formula [4]:

$$
f=\frac{1}{N \times \Delta t}
$$

where $\Delta t$ is time interval, $N$ is the number of iterations required to complete one cycle of oscillations in a fundamental mode.

As can be seen from the Equation 1, the critical damping factor is a multiple of the mass vector. This makes it easy to compare whether another damping coefficient is converging faster.

This article investigates the determination of the damping coefficient based on the oscillation of the structure in the undamped mode (according to Equation 2 with one cycle of oscillation. The procedure is a comparison with the otherwise set damping factor for a total of six computational cases (on four different structures). The efficiency of the process is evaluated based on the number of iterations.

Other parameters are considered as follows: The time interval is selected one second. The mass vector is determined based on the stiffness of each node and 
is set for each node separately (which is one of the most effective methods, for example, see [5]).

\section{Testing}

There are a total of four reference examples, which are used together for a total of six cases. The variety of examples is as large as possible to demonstrate the greatest versatility of the test procedure. Structures are modeled in 3D space and include the form-finding $(\mathrm{FF})$ and static response (SR) processes.

Cables are numerically approximated using bar (FF and SR) and cable (SR) elements, see more 6. Membranes are approximated using isoparametric triangular elements (for FF and SR), see [3] or [7].

\subsection{EXAMPLE 1}

The structure is taken from [6]. A scheme of the model structure can be seen in Figure 1. The span $L=30.48 \mathrm{~m}$. There have been many experiments on this real orthogonal cable net. Thus, numerical results can be compared with real-measured values.

Cable elements are used to model cables. All cable segments have same cross-sectional areas $1.4645 \times 10^{-4} \mathrm{~m}^{2}$ and Young's modulus $8.2737 \times 10^{10} \mathrm{~N} / \mathrm{m}^{2}$. The slack lengths of inner cables are $30.419 \mathrm{~m}$, and the remaining cables are $31.76 \mathrm{~m}$. Each of the four internal joints 4, 5, 8 and 9 carries a downward concentrated load $35.56 \mathrm{kN}$. A small self weigh of $1.46 \times 10^{-3} \mathrm{kN} / \mathrm{m}$ for cables is considered. The structure has 12 degrees of freedom. Only the static response of the design is solved.

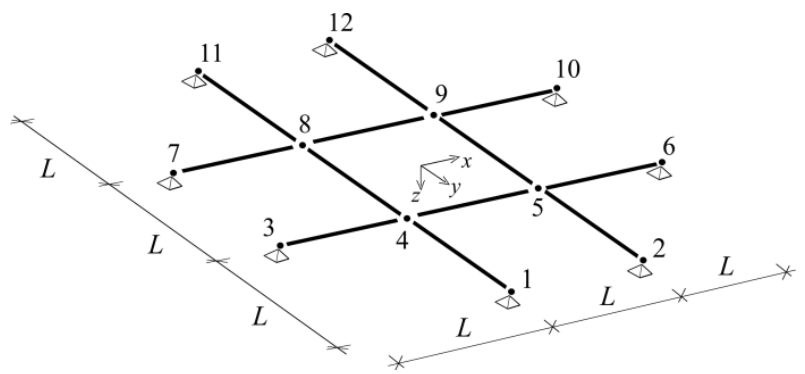

Figure 1. Schema of example 1. Perspective view.

\subsection{EXAMPLE 2}

The structure is taken from Lewis 44. The results can be compared for both processes (form-finding and static response). A structure schema is shown in Figure 2. The symmetry of initial configuration about the $x$ and $y$ centre axes is maintained. The coordinates of node 29 are $x=12 \mathrm{~m}, y=16 \mathrm{~m}$ and $z=3 \mathrm{~m}$. Bar elements are used to approximate the cables. The structure has 45 degrees of freedom.

For the form-finding process, initial geometry is generated using a pre-tension force $90 \mathrm{kN}$ in $x$ direction, (except for the centre cable where it is $120 \mathrm{kN}$ ), a $30 \mathrm{kN}$ in the $y$ direction, plus a vertically point load $4.8 \mathrm{kN}$ at all free nodes.
For static response process the cross-sectional areas is assumed to be $350 \mathrm{~mm}^{2}$ in the $x$ direction and $120 \mathrm{~mm}^{2}$ in the $y$ direction. The elastic modulus is $160 \mathrm{kN} / \mathrm{mm}^{2}$. The structure is subjected to the further increase in load $2.0 \mathrm{kN}$ at all free nodes.

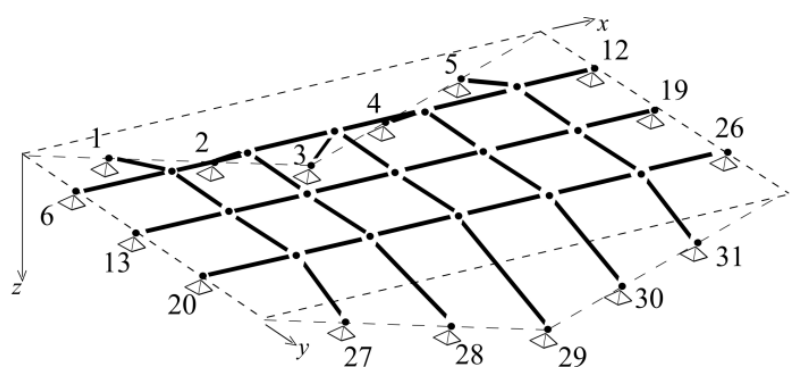

Figure 2. Schema of example 2. Perspective view.

\subsection{EXAMPLE 3}

It is a fictitious membrane structure. A scheme of the model structure can be seen in Figure 3. The structure is supported throughout the circuit and the dimensions are $L=10 \mathrm{~m}$ and $H=4 \mathrm{~m}$. To generate mesh, triangular elements with a maximum edge size of 1.25 meters are used. Thus, a model with 231 degrees of freedom is created. The model is tested for both design phases (FF and SR).

For the FF process, a constant horizontal pretension $\sigma=2000 \mathrm{kN} / \mathrm{m}^{2}$ is used (the same for directions $x$ and $y$ ). Thickness of membrane is $0.001 \mathrm{~m}$.

For a static response, a vertical flat load $f_{z}=500 \mathrm{~N} / \mathrm{m}^{2}$ is considered. The membrane parameters are: the elastic modulus is $30 \mathrm{~N} / \mathrm{mm}^{2}$, and the Poisson's ratio is 0.2 .

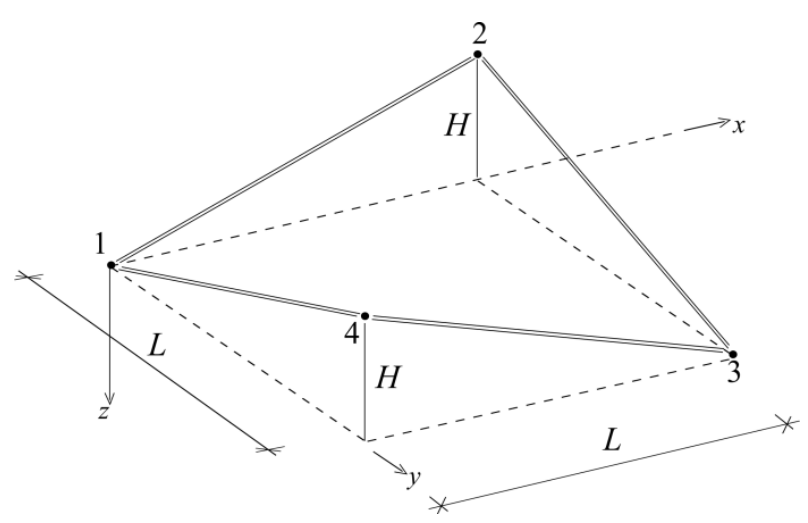

Figure 3. Schema of example 3. Perspective view.

\subsection{EXAMPLE 4}

It is a fictitious cable-membrane structure that has the parameters of a real construction. A scheme of the model structure can be seen in Figure 4 The structure is supported in nodes 1 to 6 and both top rings. The cables are around the perimeter of the structure. The dimensions of structure are $L=8 \mathrm{~m}$ and $H=3 \mathrm{~m}$ and the radius of the two top rings is $1 \mathrm{~m}$. To generate mesh, triangular elements with a 
maximum edge size of 0.7 meters are used. Thus, a model with 1623 degrees of freedom is created. The model is tested only for form-finding process.

For the FF process, a constant horizontal pretension $\sigma=1000 \mathrm{kN} / \mathrm{m}^{2}$ is used (the same for directions $x$ and $y$ ). The thickness of membrane is $0.001 \mathrm{~m}$. The horizontal pre-tension force $50 \mathrm{kN}$ is considered in all cables.

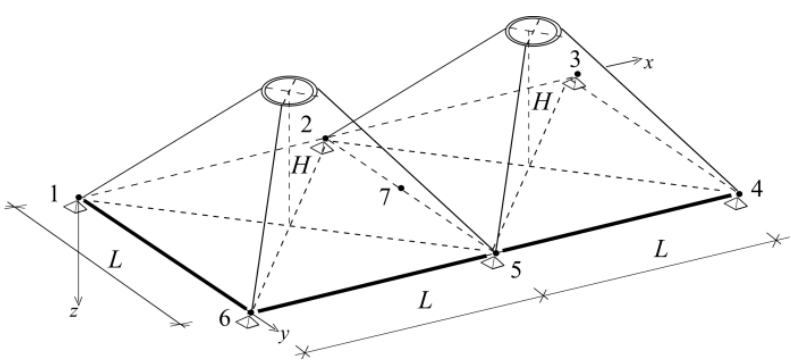

Figure 4. Schema of example 4. Perspective view.

\section{Results}

All cases were tested in the author's script for solving cable-membrane structures using the dynamic relaxation method created in MATLAB®. The numerical accuracy of all calculations was controlled by a residual limit force set at $0.0001 \mathrm{kN}$.

\subsection{EXAMPLE 1}

The numerical finite element model (in final equilibrium state) for the static response is shown in Figure 5. Fundamental frequency was estimated at $f=0.0189 \mathrm{~Hz}$, that corresponds to 0.237 multiple of the mass vector $(\alpha=0.237)$.

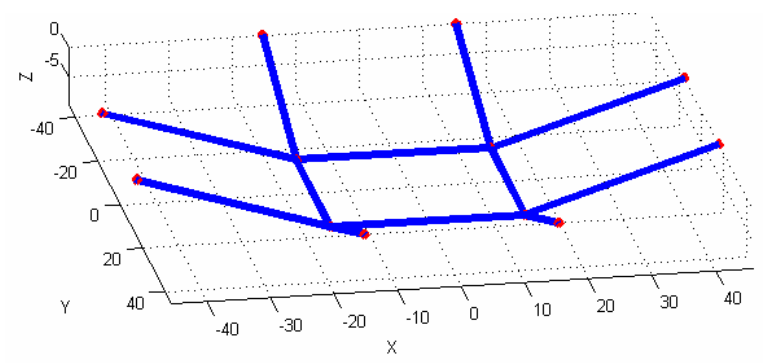

Figure 5. Example 1 (static response) in a final equilibrium state.

The Figure 6 shows the number of iterations for damping coefficient values, when $C=\alpha m$. It has been tested for $\alpha$ in the range from 0.05 to 0.6 . It can be seen that the critical damping coefficient (red square) was set very well and that the number of iterations is very minimal.

\subsection{EXAMPLE 2}

The numerical finite element model (in the initial and final state) for the form-finding process is shown in Figure 7. Fundamental frequency was estimated at

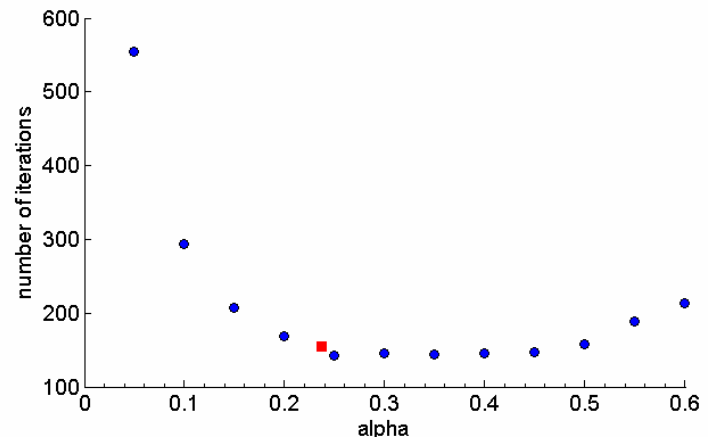

Figure 6. Comparison of the number of iterations for the damping coefficient determined according to Equations 1 and 2 (red square) with different damping coefficients for Example 1 (SR).

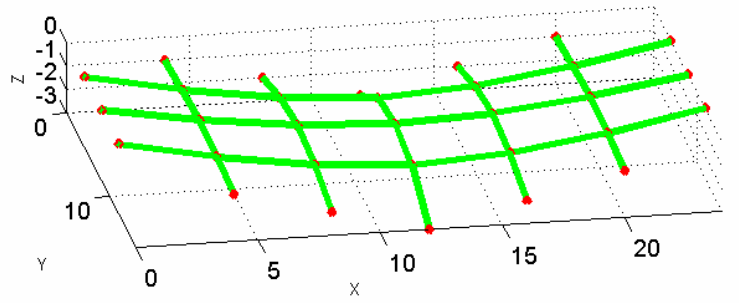

Figure 7. Example 2 (form-finding) in a final equilibrium state.

$f=0.0926 \mathrm{~Hz}$, that corresponds to 1.163 multiple of the mass vector $(\alpha=1.163)$.

The Figure 8 shows the number of iterations for damping coefficient values. It has been tested for $\alpha$ in the range from 0.8 to 1.6. It can be seen that the critical damping coefficient (red square) was set well and that the number of iterations is close to minimum.

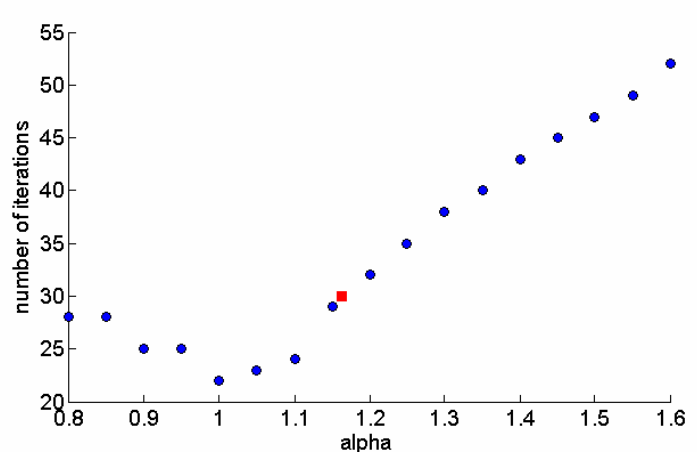

FIGURE 8. Comparison of the number of iterations for the damping coefficient determined according to Equations 1 and 2 (red square) with different damping coefficients for Example 2 (FF).

Fundamental frequency for static response was estimated at $f=0.0088 \mathrm{~Hz}$, that corresponds to 0.111 multiple of the mass vector $(\alpha=0.111)$.

The Figure 9 shows the number of iterations for damping coefficient values. It has been tested for in the range from 0.04 to 0.22 . It can be seen that the critical damping coefficient (red box) was set excellent 
and that the number of iterations is minimal.

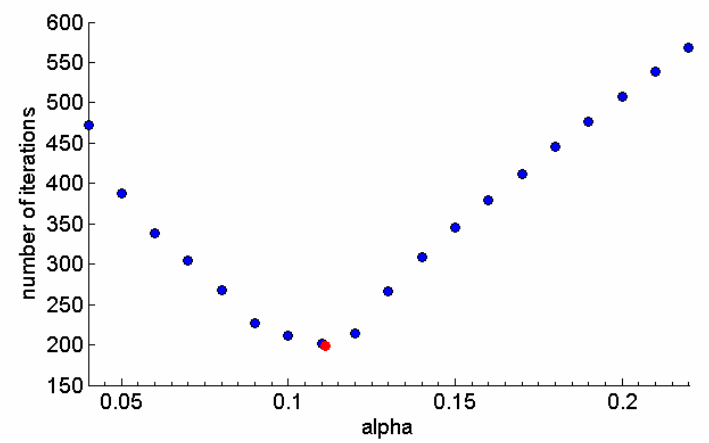

Figure 9. Comparison of the number of iterations for the damping coefficient determined according to Equations 1 and 2 (red square) with different damping coefficients for Example 2 (SR).

\subsection{ExAmple 3}

The numerical finite element model (in the initial and final state) for the form-finding process is shown in Figure 10. Fundamental frequency was estimated at $f=0.0151 \mathrm{~Hz}$, that corresponds to 0.190 multiple of the mass vector $(\alpha=0.190)$.

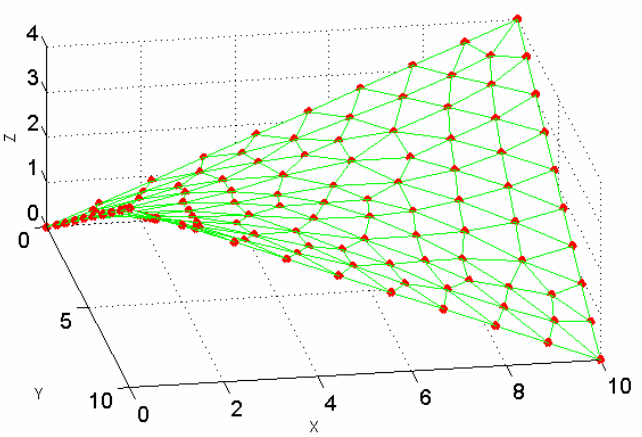

Figure 10. Example 3 (form-finding) in a final equilibrium state.

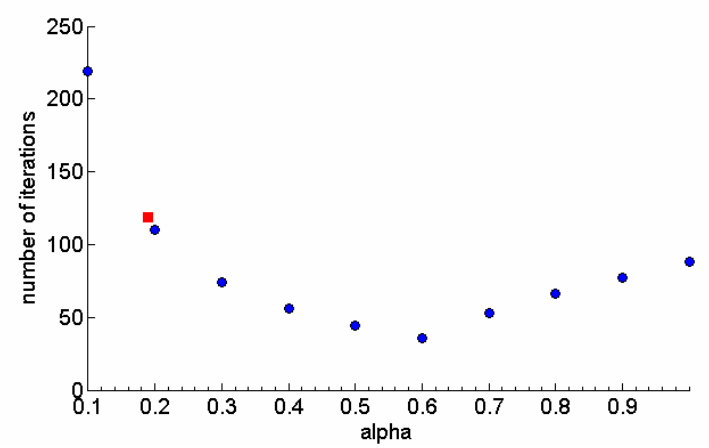

Figure 11. Comparison of the number of iterations for the damping coefficient determined according to Equations 1 and 2 (red square) with different damping coefficients for Example 3 (FF).

The Figure 11 shows the number of iterations for damping coefficient values. It has been tested for in the range from 0.1 to 1.0 . It can be seen that the critical damping coefficient (red square) was not set well and that the number of iterations is not close to minimum.

Fundamental frequency for static response was estimated at $f=0.0077 \mathrm{~Hz}$, that corresponds to 0.096 multiple of the mass vector $(\alpha=0.096)$.

The Figure 12 shows the number of iterations for damping coefficient values. It has been tested for in the range from 0.04 to 0.22 . It can be seen that the critical damping coefficient (red square) was set excellent and that the number of iterations is minimal.

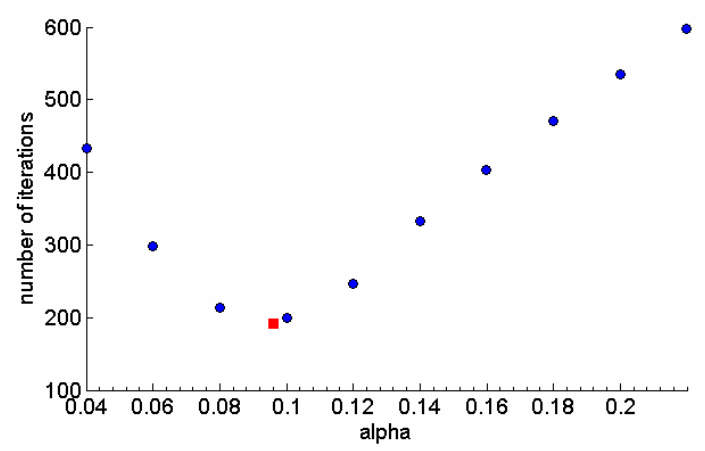

Figure 12. Comparison of the number of iterations for the damping coefficient determined according to 1 and 2 (red square) with different damping coefficients for Example 3 (SR).

\subsection{EXAMPLE 4}

The numerical finite element model (in the initial and final state) for the form-finding process is shown in Figure 13. Fundamental frequency was estimated at $f=0.0417 \mathrm{~Hz}$, that corresponds to 0.523 multiple of the mass vector $(\alpha=0.523)$.

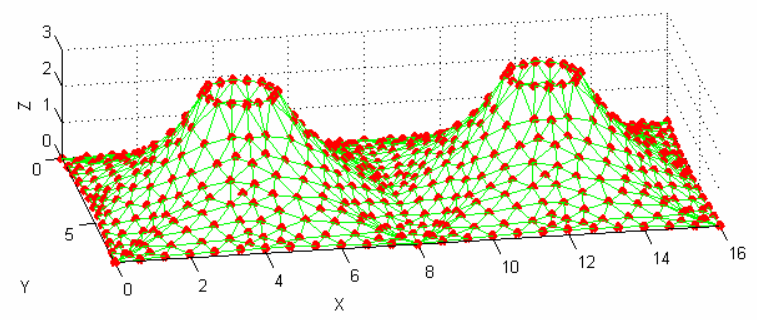

Figure 13. Comparison of the number of iterations for the damping coefficient determined according to 1 and 2 (red square) with different damping coefficients for Example 4 (FF).

The Figure 14 shows the number of iterations for damping coefficient values. It has been tested for $\alpha$ in the range from 0.2 to 0.9 . It can be seen that the critical damping coefficient (red box was set well and that the number of iterations is close to minimum.

\section{Conclusions}

This article investigated the determination of the damping coefficient based on oscillation of the struc- 


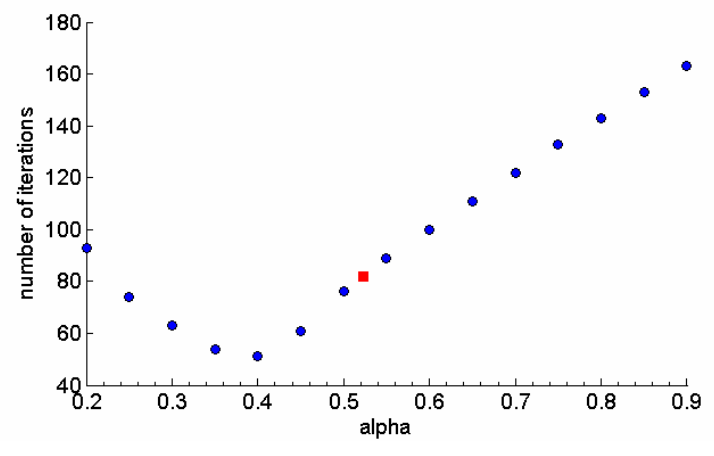

Figure 14. Comparison of the number of iterations for the damping coefficient determined according to 1 and 2 (red square) with different damping coefficients for Example $4(\mathrm{FF})$.

ture in the undamped mode with one cycle of oscillation. The procedure was a comparison with the otherwise set damping factor for a total of six computational cases (on four different structures). The efficiency of the process was evaluated based on the number of iterations.

Base on the results presented in previous section, it can be concluded that the proposed method of determining the dynamic relaxation damping coefficient is very suitable for cable-membrane structures. When setting a damping coefficient according to the proposed procedure, the minimum number of iterations (or near to minimum) was achieved in four of the six test cases. Excellent results are achieved by the static response analysis procedure with a construction close to the final state.

It has been shown that only one oscillation cycle can be used to determine the fundamental frequency. Using multiple cycles would probably improve the frequency estimate, but the CPU time would increase. It could be tested in further studies.

\section{LIST OF SYMBOLS}

$C$ viscous damping coefficient $\left[\mathrm{ts}^{-1}\right]$

$m$ mass (or vector of masses) [t]

$f$ fundamental frequency of oscillation $[\mathrm{Hz}]$

$\Delta t$ time interval $[\mathrm{s}]$

$N$ the number of iterations required to complete one cycle of oscillations in a fundamental mode $[-]$

$L$ the span of the structure $[\mathrm{m}]$

$H$ the height of the structure [m]

$\alpha$ multiple of the mass vector $[-]$

\section{ACKNowledGEMENTS}

The results presented in this paper are outputs of the research project "SGS18/037/OHK1/1T/11- Development and application of advanced algorithms for numerical analysis and modelling in mechanics of structures and materials" supported by the Czech Technical University in Prague.

\section{REFERENCES}

[1] K. Linkwitz, H. J. Schek. A new method of analysis of prestressed cable networks and its use on the roofs of the Olympic game facilities at Munich. IABSE, 1972.

[2] E. R. Bletzinger. A general finite element approach to the form finding of tensile structures by the updated reference strategy. Int J Space Struct (14):131-146, 1999.

[3] B. H. V. Topping, P. Iványi. Computer Aided Design of Cable Membrane Structures. Saxe-Coburg Publications, 2007.

[4] W. J. Lewis. Tension structures: form and behaviour. Thomas Telford, 2003.

[5] M. Huttner, J. Maca, P. Fajman. The efficiency of dynamic relaxation methods in static analysis of cable structures. Advances in Engineering Software (89):28-35, 2015.

[6] H. Deng, Q. F. Jiang, A. S. K. Kwan. Shape finding of incomplete cable-strut assemblies containing slack and prestressed elements. Computers and Structures (83):21-22, 1767-1779, 2005.

[7] M. R. Barnes. Form and stress engineering of tension structures. Structural Engineering Review (6 (3-4)):175-202, 1994. 